Review began 02/01/2022 Review ended 02/15/2022 Published 02/18/2022

() Copyright 2022

Nagata et al. This is an open access article distributed under the terms of the Creative Commons Attribution License CC-BY 4.0., which permits unrestricted use, distribution, and reproduction in any medium, provided the original author and source are credited.

\section{Long-Term Survival of a Patient With Peritoneal Carcinomatosis From Pancreatic Cancer Maintained by Nanoliposomal Irinotecan as Third-Line Chemotherapy}

\author{
Keiji Nagata ${ }^{1}$, Masatoshi Kajiwara ${ }^{2,1}$, Takahisa Fujikawa ${ }^{1}$
}

1. Surgery, Kokura Memorial Hospital, Kitakyushu, JPN 2. Gastroenterological Surgery, Fukuoka University Hospital, Fukuoka, JPN

Corresponding author: Takahisa Fujikawa, takamaminao@gmail.com

\begin{abstract}
Pancreatic cancer is still one of the most fatal neoplastic diseases, and the recurrence occurs in more than $80 \%$ of the patients even though radical resection is performed. We experienced a long-term survival case of a patient with peritoneal carcinomatosis from pancreatic cancer maintained by nanoliposomal irinotecan (nal-IRI) in combination with fluorouracil and folinic acid (FF) as third-line chemotherapy. Nal-IRI + FF combination chemotherapy is one of the promising options for the management of intractable recurrent pancreatic cancer.
\end{abstract}

Categories: Gastroenterology, General Surgery, Oncology

Keywords: long-term survival, combination chemotherapy, nasoliposomal irinotecan, carcinomatosa peritoni, pancreatic cancer

\title{
Introduction
}

Pancreatic cancer is still one of the most fatal diseases. Surgical resection is the only treatment that can be expected to cure pancreatic cancer, but the proportion of resectable cases is about $15 \%-20 \%$ [1,2].

Furthermore, there are many cases of recurrence even after radical resection. Chemotherapy is currently the main treatment for pancreatic cancer with distant metastasis, and nanoliposomal irinotecan (nal-IRI) is the first drug delivery system (DDS) preparation to be used for pancreatic cancer.

We report a case of peritoneal dissemination and abdominal wall metastasis from pancreatic cancer in which nal-IRI + fluorouracil and folinic acid (FF) combination therapy was effective as third-line chemotherapy and the disease could be controlled for a long period of time.

\section{Case Presentation}

A 70-year-old female presented with abdominal pain, and she was diagnosed with pancreatic tail cancer by computed tomography (CT) scan, magnetic resonance cholangiopancreatography (MRCP), and endoscopic retrograde cholangiopancreatography/endoscopic ultrasound (ERCP/EUS) (Figure 1, Panel a). She underwent radical distal pancreatectomy with regional lymph node dissection and revealed moderately differentiated invasive ductal carcinoma accompanied by regional lymph node involvement (Figure 1, Panel b). 


\section{Cureus}

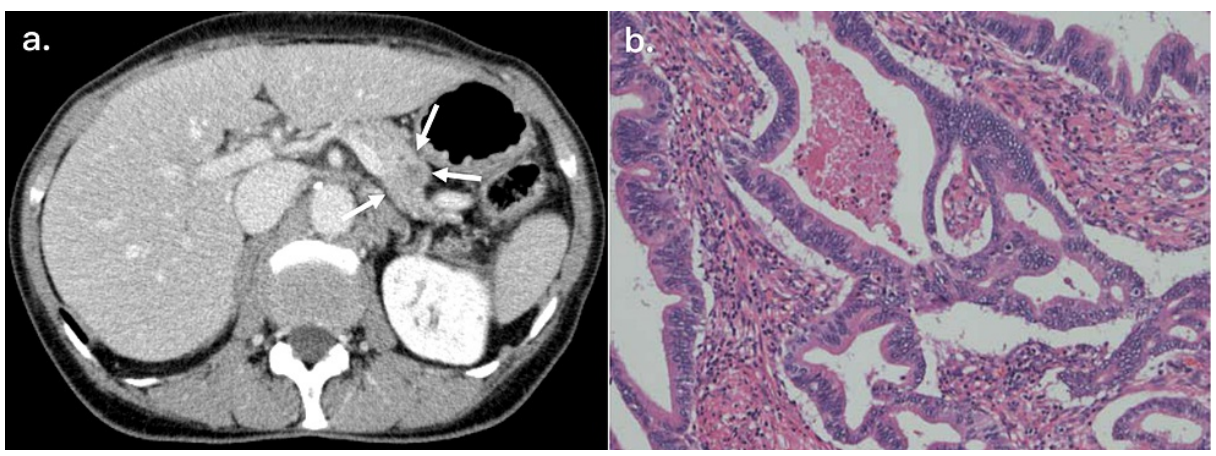

\section{FIGURE 1: Tumor characteristics of the current case}

(a) Preoperative CT findings. Abdominal enhanced CT scan showed a hypovascular mass (arrows), approximately $22 \mathrm{~mm}$ in diameter, at the pancreatic tail, and an invasion to the splenic artery and vein was suspected. (b) Microscopic examination revealed moderately differentiated invasive ductal carcinoma of the pancreas accompanied by regional lymph node involvement.

CT: Computed tomography.

The postoperative course was uneventful, and the patient recovered without any complications.

She subsequently received six-month adjuvant S-1 chemotherapy (six weeks/cycle, $80 \mathrm{mg} /$ day of oral S-1 on days 1-28), and the stable disease-free status was maintained, although multiple intraperitoneal and abdominal wall masses were developed nine months after tumor resection (Figure 2).
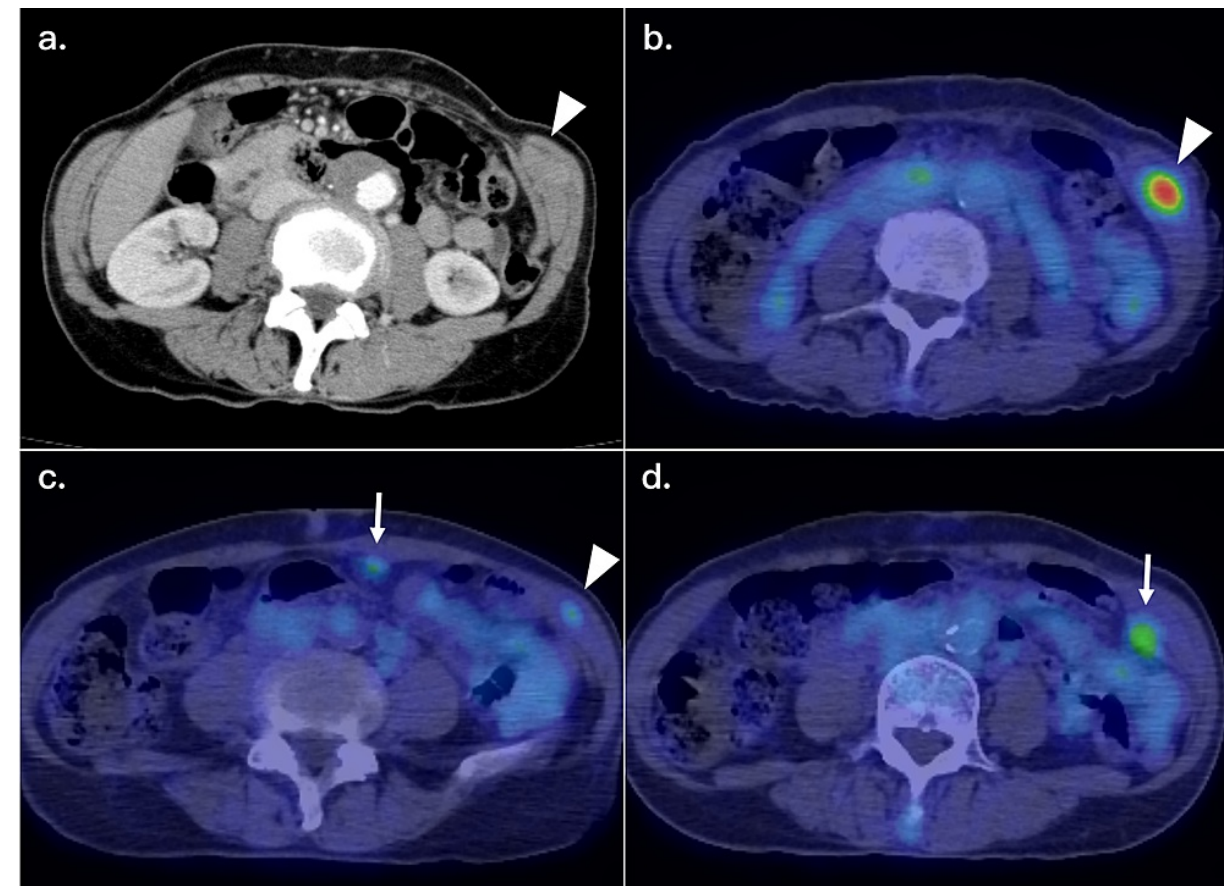

\section{FIGURE 2: CT and PET-CT findings at the first recurrence}

(a, b) An enhanced CT scan showed a contrasted nodule in the left abdominal wall, with positive FDG uptake (SUVmax $=6.52$ ). (c, d) PET-CT also showed multiple intraperitoneal and abdominal wall metastases with FDG uptake at the peritoneum (arrows) and in the abdominal wall (arrowheads).

CT: Computed tomography; PET-CT: positron emission tomography-computed tomography; FDG: fluorodeoxyglucose; SUVmax: maximum standardized uptake value.

The whole clinical course of the current patient with serial changes in serum duke pancreatic monoclonal antigen type-2 (DUPAN-2) values was summarized in Figure 3. 


\section{Cureus}

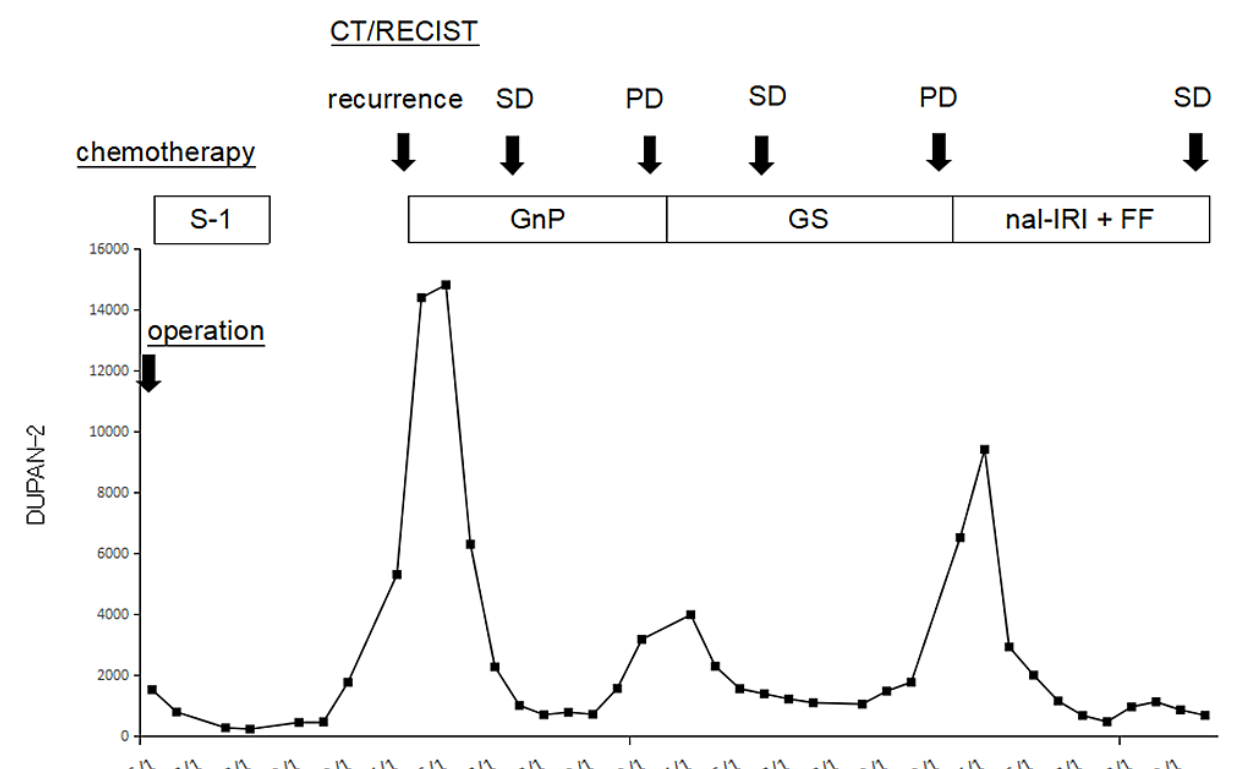

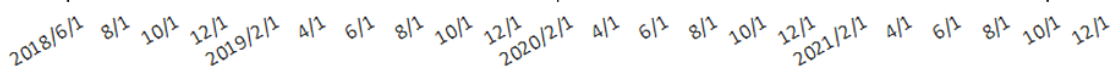

\section{FIGURE 3: Clinical course of the current patient with pancreatic tail} cancer

The patient received six-month adjuvant S-1 chemotherapy after distal pancreatectomy. Multiple intraperitoneal and abdominal wall masses were developed nine months after tumor resection. First-line chemotherapy (GnP) was introduced, and her disease condition was SD for 12 months, but it later became PD. Second-line chemotherapy (GS) was introduced, and SD was maintained but became PD 11 months later. Third-line chemotherapy (nal-IRI + FF) was then initiated, and her tumor condition was maintained SD with a significant reduction of DUPAN-2 values for 12 months.

GnP: Gemcitabine plus nab-paclitaxel; SD: stable disease; PD: progressive disease; GS: gemcitabine plus S-1 nal-IRI + FF: nanoliposomal irinotecan plus fluorouracil and folinic acid; RECIST: response evaluation criteria in solid tumors; DUPAN-2: duke pancreatic monoclonal antigen type-2; CT: computed tomography.

First-line chemotherapy using gemcitabine plus nab-paclitaxel (GnP) (four weeks/cycle, $1000 \mathrm{mg} / \mathrm{m}^{2}[1200$ $\mathrm{mg}$ ] of gemcitabine plus $125 \mathrm{mg} / \mathrm{m}^{2}$ [150 mg] of nab-paclitaxel at days 1, 8, and 15) was introduced, and her disease condition was stable for 12 months but later became progressive (Figure 4, Panel a). Second-line chemotherapy using gemcitabine plus S-1 (GS) (three weeks/cycle, $80 \mathrm{mg} /$ day of oral S-1 on days 1-14 plus $1000 \mathrm{mg} / \mathrm{m}^{2}$ [1200 mg] of gemcitabine at days 1 and 8 ) was introduced, and stable tumor condition was maintained (Figure 4, Panel b), but the tumors became progressive 11 months thereafter (Figure 4, Panel c). 


\section{Cureus}
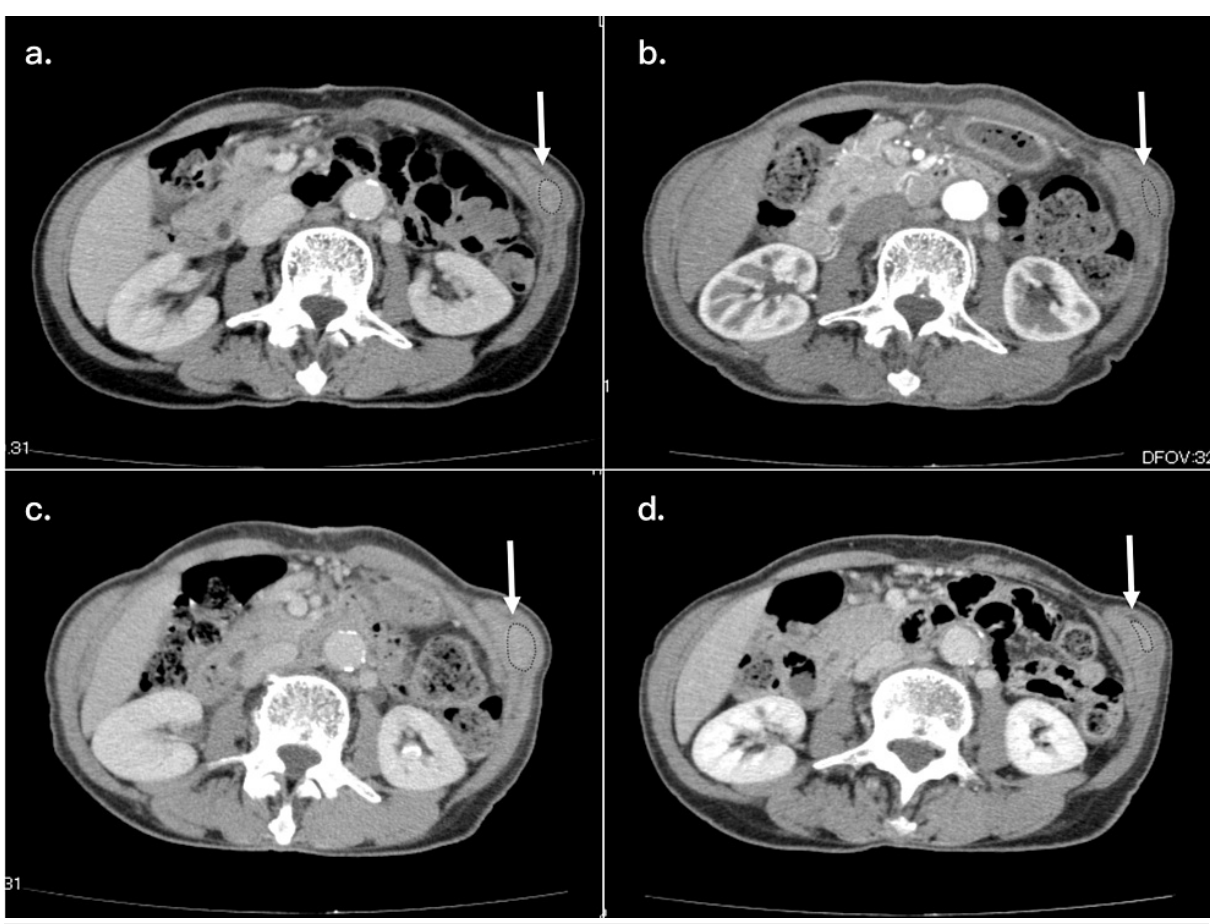

FIGURE 4: Sequential CT findings of the recurrent abdominal wall tumor (arrows and dotted areas)

(a) The tumor in the left lateral abdominal wall was initially maintained as SD for 12 months after starting first-line chemotherapy but later became PD. The tumor was maintained as SD for 11 months after the introduction of second-line chemotherapy (b) but became PD thereafter (c). (d) Subsequently, third-line chemotherapy using nalIRI + FF was introduced, and the tumor was maintained as SD for 12 months with an excellent physical condition.

CT: Computed tomography; SD: stable disease; PD: progressive disease; nal-IRI + FF: nanoliposomal irinotecan plus fluorouracil and folinic acid.

Third-line chemotherapy was then scheduled using nal-IRI in combination with FF, and she was well tolerated without any severe adverse effects. Her height, body weight, and body mass index were $147.6 \mathrm{~cm}$, $36.5 \mathrm{~kg}$, and $16.8 \mathrm{~kg} / \mathrm{m}^{2}$, respectively. She received $50 \mathrm{mg} / \mathrm{m}^{2}(60 \mathrm{mg})$ of nal-IRI plus $2400 \mathrm{mg} / \mathrm{m}^{2}(3000 \mathrm{mg})$ of fluorouracil and $200 \mathrm{mg} / \mathrm{m}^{2}(250 \mathrm{mg}$ ) of folinic acid every two weeks. The dose of nal-IRI was reduced to $50 \mathrm{mg} / \mathrm{m}^{2}$ because she was homozygous for UGT1A1*6 (uridine diphosphate glucuronosyltransferase 1A1 gene) by UGT1A1 genetic polymorphism analysis. Her tumor condition is currently maintained as a stable disease with a significant reduction of DUPAN-2 values, and it remains stable without tumor progression for 12 months (47 months after initial tumor resection) (Figure 4, Panel d).

\section{Discussion}

Pancreatic cancer is still one of the most deadly cancers with a five-year survival rate of less than $20 \%$ even after curative resection [3,4]. Median survival for patients receiving surgical resection is only 12.6 months, and the high recurrence rate is the main reason for the very poor prognosis [5]. Recurrence and/or metastasis from pancreatic cancer is considered to present as multiple recurrent lesions or peritoneal dissemination $[6,7]$, although some selected patients may have a chance of long-term survival, thanks to recent advances in combination chemotherapy $[8,9]$.

As second-line chemotherapy after FOLFIRINOX (a combination of fluorouracil, leucovorin, irinotecan, and oxaliplatin) therapy or GnP therapy, nal-IRI was approved and launched for pancreatic cancer with distant metastasis. Irinotecan is hydrolyzed to the active metabolite $\mathrm{SN}-38$ by carboxylesterase in vivo and inhibits DNA synthesis through inhibition of type I topoisomerase [10]. Nal-IRI is a nanoliposomal preparation of irinotecan modified with polyethylene glycol and has been reported to have the following effects: (1) increased tumor accumulation due to increased vascular permeability and retention [11] and (2) enhanced antitumor activity by prolonging the duration of SN-38 exposure within the tumor [12].

Nal-IRI + FF therapy showed a significant improvement in overall survival (OS) in phase III clinical trial (NAPOLI-1 study) compared to the FF group (median survival for nal-IRI + FF group: 6.1 months versus for FF group: 4.2 months, $\mathrm{p}=0.012$ ) [13]. The nal-IRI + FF combination therapy is considered to be a promising regimen as a new second-line chemotherapy option for metastatic pancreatic cancer that has progressed 
after gemcitabine-related regimens. Assi et al. [14] reported a long-term survival case with nal-IRI as second-line chemotherapy. In his paper, the patient received 58 cycles of nal-IRI + FF totally, which she tolerated very well without any dose adjustments until imaging showed evidence of PD. Relative to the nal-IRI + FF initiation date, the OS was 40 months. However, to the best of our knowledge, this is a rare case report that shows nal-IRI + FF therapy has been successful as third-line chemotherapy for metastatic pancreatic cancer and long-term disease control has been obtained.

In the current case, GnP therapy and GS therapy were performed as first-line and second-line chemotherapy for peritoneal dissemination and abdominal wall recurrence of pancreatic cancer, respectively, but the tumor condition became PD. Subsequently, nal-IRI + FF therapy was started as third-line chemotherapy, resulting in a significant reduction of DUPAN-2 levels, reduced size of the intraperitoneal and abdominal wall lesions, and achievement of long-term disease control. The tumor status of SD is still maintained as 12 months after the start of nal-IRI + FF therapy (47 months after tumor resection).

Glassman et al. [15] demonstrated the safety and effectiveness of nal-IRI + FF therapy in advanced pancreatic cancer patients. In his study, dose reductions were most frequently due to fatigue and diarrhea but were not associated with worse outcomes. In the present case, the patient received 20 cycles of nal-IRI + FF therapy and tolerated the regimen without any dose reductions. Her adverse effects included nausea and fatigue, all of which were managed with medications. Since nal-IRI + FF is still effective, further follow-up is necessary to determine the efficacy and tolerability of nal-IRI in the longer term. Nevertheless, the predictors of the effects of nal-IRI therapy are still unclear, and analysis by accumulating future cases is necessary.

\section{Conclusions}

We reported a long-term survival case of a patient with peritoneal carcinomatosis from pancreatic cancer. After the failure of first-line and second-line gemcitabine-based chemotherapy, the patient was maintained with a stable tumor condition by nal-IRI + FF therapy as third-line chemotherapy for an additional 12 months. So, nal-IRI + FF combination chemotherapy is one of the promising options for the management of intractable recurrent pancreatic cancer.

\section{Additional Information \\ Disclosures}

Human subjects: Consent was obtained or waived by all participants in this study. Conflicts of interest: In compliance with the ICMJE uniform disclosure form, all authors declare the following: Payment/services info: All authors have declared that no financial support was received from any organization for the submitted work. Financial relationships: All authors have declared that they have no financial relationships at present or within the previous three years with any organizations that might have an interest in the submitted work. Other relationships: All authors have declared that there are no other relationships or activities that could appear to have influenced the submitted work.

\section{References}

1. Vincent A, Herman J, Schulick R, Hruban RH, Goggins M: Pancreatic cancer. Lancet. 2011, 378:607-20. 10.1016/S0140-6736(10)62307-0

2. Sharma C, Eltawil KM, Renfrew PD, Walsh MJ, Molinari M: Advances in diagnosis, treatment and palliation of pancreatic carcinoma: 1990-2010. World J Gastroenterol. 2011, 17:867-97. 10.3748/wjg.v17.i7.867

3. Schnelldorfer T, Ware AL, Sarr MG, et al.: Long-term survival after pancreatoduodenectomy for pancreatic adenocarcinoma: is cure possible?. Ann Surg. 2008, 247:456-62. 10.1097/SLA.0b013e3181613142

4. Hsu CC, Herman JM, Corsini MM, et al.: Adjuvant chemoradiation for pancreatic adenocarcinoma: the Johns Hopkins Hospital-Mayo Clinic collaborative study. Ann Surg Oncol. 2010, 17:981-90. 10.1245/s10434-0090743-7

5. Bilimoria KY, Bentrem DJ, Ko CY, Ritchey J, Stewart AK, Winchester DP, Talamonti MS: Validation of the 6th edition AJCC pancreatic cancer staging system: report from the National Cancer Database. Cancer. 2007, 110:738-44. 10.1002/cncr.22852

6. Tonini V, Zanni M: Pancreatic cancer in 2021: what you need to know to win . World J Gastroenterol. 2021, 27:5851-89. 10.3748/wjg.v27.i35.5851

7. Hishinuma S, Ogata Y, Tomikawa M, Ozawa I, Hirabayashi K, Igarashi S: Patterns of recurrence after curative resection of pancreatic cancer, based on autopsy findings. J Gastrointest Surg. 2006, 10:511-8. 10.1016/j.gassur.2005.09.016

8. Okamoto Y, Maeba T, Kakinoki K, et al.: A patient with unresectable advanced pancreatic cancer achieving long-term survival with gemcitabine chemotherapy. World J Gastroenterol. 2008, 14:6876-80. 10.3748/wjg. 14.6876

9. Sumiyoshi T, Shima Y, Okabayashi T, et al.: Long-term survival following pancreatectomy and s-1 chemotherapy for pancreatic acinar cell carcinoma with peritoneal dissemination: a case report and literature review. Medicine (Baltimore). 2015, 94:e378. 10.1097/MD.0000000000000378

10. Kawato Y, Aonuma M, Hirota Y, Kuga H, Sato K: Intracellular roles of SN-38, a metabolite of the camptothecin derivative CPT-11, in the antitumor effect of CPT-11. Cancer Res. 1991, 51:4187-91.

11. Drummond DC, Noble CO, Guo Z, Hong K, Park JW, Kirpotin DB: Development of a highly active 


\section{Cureus}

nanoliposomal irinotecan using a novel intraliposomal stabilization strategy. Cancer Res. 2006, 66:3271-7. 10.1158/0008-5472.CAN-05-4007

12. Kalra AV, Kim J, Klinz SG, et al.: Preclinical activity of nanoliposomal irinotecan is governed by tumor deposition and intratumor prodrug conversion. Cancer Res. 2014, 74:7003-13. 10.1158/0008-5472.CAN-140572

13. Wang-Gillam A, Li CP, Bodoky G, et al.: Nanoliposomal irinotecan with fluorouracil and folinic acid in metastatic pancreatic cancer after previous gemcitabine-based therapy (NAPOLI-1): a global, randomised, open-label, phase 3 trial. Lancet. 2016, 387:545-57. 10.1016/S0140-6736(15)00986-1

14. Assi HA, Shikdar S, Alhyari L, Aljumaily R: Long-term survival with nanoliposomal irinotecan in pancreatic cancer. Pancreas. 2020, 49:e95-6. 10.1097/MPA.0000000000001649

15. Glassman DC, Palmaira RL, Covington CM, et al.: Nanoliposomal irinotecan with fluorouracil for the treatment of advanced pancreatic cancer, a single institution experience. BMC Cancer. 2018, 18:693. 10.1186/s12885-018-4605-1 